\title{
PRELIMINARY REPORT ON PREDICTION OF SPINAL CORD ISCHEMIA IN ENDOVASCULAR STENT GRAFT REPAIR OF THORACIC AORTIC ANEURYSM BY RETRIEVABLE STENT GRAFT
}

Shin Ishimaru, MD

Satoshi Kawaguchi, MD

Nobusato Koizumi, MD

Yukio Obitsu, MD

Mikio Ishikawa, MD
Objective: To predict spinal cord ischemia after endovascular stent graft repair of descending thoracic aortic aneurysms, temporary interruption of the intercostal arteries (including the aneurysm) was performed by placement of a novel retrievable stent graft (Retriever) in the aorta under evoked spinal cord potential monitoring. Methods: From February 1995 to October 1997, endovascular stent graft repair of descending thoracic aortic aneurysms was performed in 49 patients after informed consent was obtained. In 16 patients with aneurysms located in the middle and distal segment of the descending aorta, the Retriever was placed temporarily before stent graft deployment. The Retriever consisted of two units of self-expanding zigzag stents connected in tandem with stainless steel struts. Each strut was collected in a bundle fixed to a pushing rod, and the stent framework was lined with an expanded polytetrafluoroethylene sheet. The Retriever was delivered beyond the aneurysm through a sheath and was retracted into the sheath 20 minutes later. A stent graft for permanent use was deployed in patients whose predeployment test results with the Retriever were favorable. Evoked spinal cord potential was monitored throughout placement of the Retriever and stent grafting until the next day. Results: The Retriever was placed in 17 aneurysms in 16 patients. There were no changes in amplitude or latency of evoked spinal cord potential records obtained before or during Retriever placement. After withdrawal of the Retriever, all aneurysms were excluded from circulation immediately after permanent stent grafting. There were no changes in evoked spinal cord potential, nor were neurologic deficits seen after stent graft deployment in any patient. Conclusions: These results suggest that predeployment testing with the Retriever under evoked spinal cord potential monitoring is promising as a predictor of spinal cord ischemia in candidates for stent graft repair of thoracic aortic aneurysms. (J Thorac Cardiovasc Surg 1998;115:811-8)
$B_{\mathrm{p}}^{\mathrm{c}}$ ecause of the increase in the number of elderly persons, there has been a great growth in cardiovascular diseases associated with arteriosclerosis, drastically increasing the need for prevention and treatment. Thoracic aortic aneurysm, which is generally caused by sclerotic degeneration, is a lifethreatening disease. Surgical replacement of the

The Second Department of Surgery, Tokyo Medical College, Tokyo, Japan.

Received for publication Feb. 28, 1997; revisions requested May 12, 1997; revisions received Nov. 14, 1997; accepted for publication Nov. 14, 1997.

Address for reprints: Shin Ishimaru, MD, the Second Department of Surgery, Tokyo Medical College, 6-7-1 Nishishinjuku, Shinjuku-ku, Tokyo 160, Japan.

Copyright (C) 1998 by Mosby, Inc.

0022-5223/98 $\$ 5.00+0 \quad \mathbf{1 2 / 1 / 8 7 6 8 6}$ aorta with a prosthetic vascular graft followed by resection of the aneurysm is the most common procedure to prevent fatal rupture, because the long-term prognosis of conservatively treated patients is poor. Although there have been remarkable improvements in the results of surgery for descending thoracic aortic aneurysm as a result of technical advances, progress in assist procedures, and advanced prosthetic grafts, the operative mortality rate remains $4 \%$ to $12 \%$, even at institutions with extensive experience. ${ }^{1,2}$ It is clear that unsatisfactory results of aortic surgery can be caused by organ malfunction (cerebrovascular, coronary, or renal artery disturbances) associated with arteriosclerosis. Furthermore, other inflammatory diseases involving the aorta present unavoidable surgical risks for aneurysm management. Consequently, minimally 


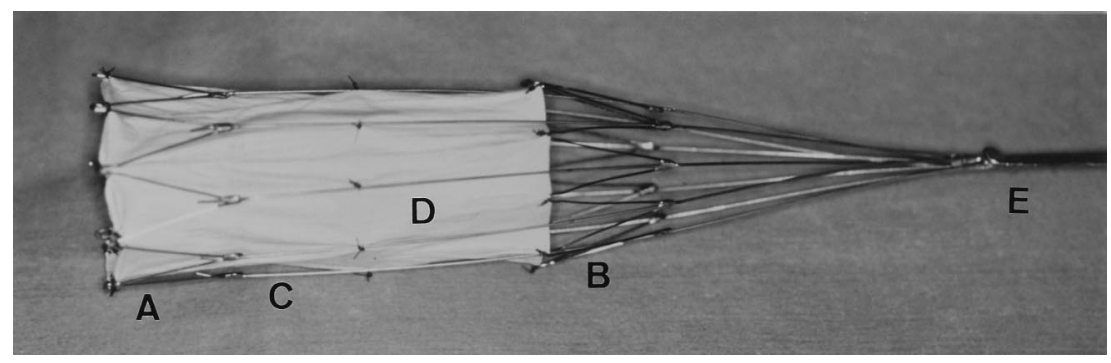

Fig. 1. The expanded Retriever. $A$, Proximal set of $Z$ stent; $B$, distal set of $Z$ stent; $C$, strut; $D$, expanded PTFE sheet; $E$, pushing rod.

invasive surgery is expected to enable successful treatment of patients at high risk.

Since the first clinical experience of endovascular stent graft repair of abdominal aortic aneurysm was reported by Parodi and colleagues ${ }^{3}$ in 1991, endovascular surgical treatment of aortic aneurysms with stent grafts has attracted attention as a minimally invasive alternative to conventional open operation. In 1992, catheter-based stent graft repair of descending thoracic aortic aneurysm was performed by the Stanford group. ${ }^{4}$ They used a self-expanding V-shaped stainless-steel stent covered with woven polyester fabric graft that was delivered to the target region through a $26 \mathrm{~F}$ polytetrafluoroethylene (PTFE) delivery sheath catheter placed in the lumen of the aneurysm. Decompression of the aneurysmal sac and reconstruction of the aorta were simultaneously achieved immediately after deployment of the stent graft, which expanded itself at both the proximal and distal necks of the aneurysm. Mitchell and associates ${ }^{5}$ reported complete thrombotic sealing in 39 of 44 thoracic aortic aneurysms $(88 \%)$ operated on during a 34-month period. However, there are several unsolved issues concerning not only serious anatomic circumstances, such as sclerotic occlusive changes or tortuosity of the aortofemoral route for delivery devices and characteristic curves present in the distal aortic arch where meticulously precise deployment of the stent graft is essential, but also concerning the occlusion as a result of stent graft deployment of tributaries for the spinal column located near the aneurysm. In particular, stent grafts tend to overlie intercostal arteries in aneurysms of the middle and distal descending aorta, consequently inducing spinal cord ischemia and occasionally resulting in paraplegia.

The purpose of this clinical study was to try to predict spinal cord ischemia after endovascular stent graft repair of descending thoracic aortic aneurysms, especially those located in the middle and distal segment. Evoked spinal cord potential (ESCP) monitoring was used during temporary interruption of intercostal arteries including the aneurysm by placement of a novel retrievable stent graft device, the Retriever.

\section{Patients and methods}

After approval of the Ethics Committee of Tokyo Medical College and informed consent were obtained, endovascular stent graft repair of descending thoracic aortic aneurysm was performed on 49 patients between February 1995 and October 1997. There were 44 men and five women with an average age of 68 years (range 23 to 78 years). Morphologic studies revealed true aneurysm in 23 patients, chronic aortic dissection in 18 patients, and pseudoaneurysm in eight patients (four inflammatory and four traumatic). In 16 patients with aneurysm located either at T- 8 or below or that in T- 6 or below extending over T-8 (nine true, two inflammatory, and five dissection), a novel retrievable stent graft, the Retriever, was placed temporarily (for at least 20 minutes) under ESCP monitoring before permanent stent graft deployment.

Our retrievable stent graft was developed by a modification of the self-expanding stainless-steel Gianturco Z stent. ${ }^{6}$ All wires were $0.4 \mathrm{~mm}$ stainless steel (Fig. 1). The basic framework of the sea anchor-shaped stent consisted of nine stainless longitudinal steel struts connected at two sections by two sets of cylindric zigzag self-expanding $V$-shaped wires with nine bends per set. The length of the V-shaped wires was $25 \mathrm{~mm}$ and the expanded diameter varied from 30 to $36 \mathrm{~mm}$. Lining fabric extended from the distal tips of the proximal set of wires, covering the $55 \mathrm{~mm}$ interval between the two bands of zigzag steel wire (the portion of the struts connecting the proximal and distal sets of wires) and the $25 \mathrm{~mm}$ distance to the tips of the distal set of V-shaped wires. The overall length of the fabric was $80 \mathrm{~mm}$. The entire structure was collected in a bundle and connected to the pushing rod. The stent fabric, expanded PTFE sheet* $0.1 \mathrm{~mm}$ in thickness, was attached by 5-0 polypropylene sutures.

\footnotetext{
*Gore-Tex sheet, registered trademark of W. L. Gore \& Associates, Inc., Newark, Del.
} 

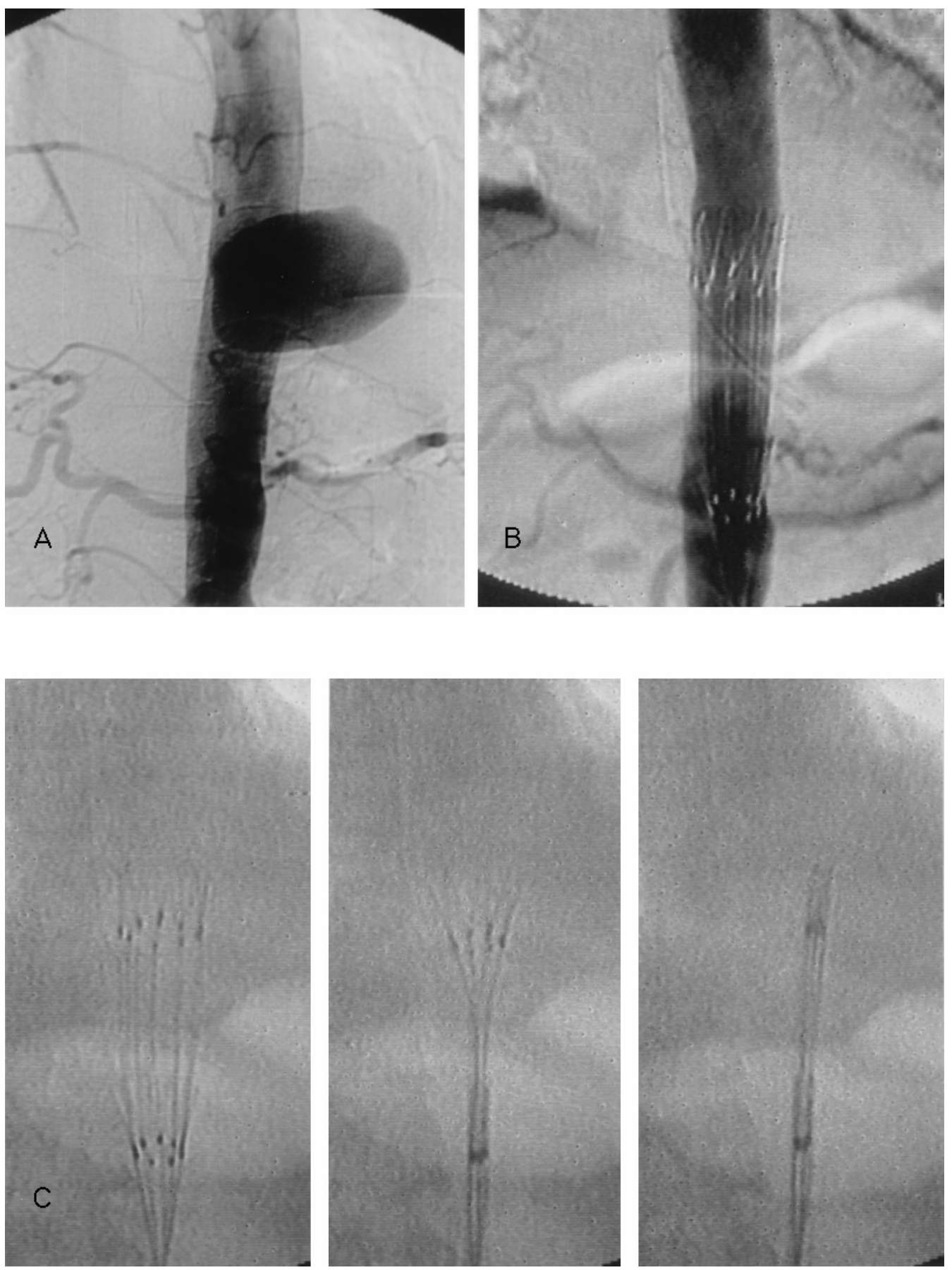

Fig. 2. A, Digital subtraction angiography of nonspecific inflammatory aneurysm located in the distal segments of the descending thoracic aorta in patient 2. B, Retriever placed through the aneurysm extending above and below it. $\mathbf{C}$, Retriever retracted into a sheath after predeployment test for 21 minutes.

With the patient under general anesthesia, the femoral artery was surgically exposed. After administration of heparin at 50 to $100 \mathrm{U} / \mathrm{kg}$ body weight, a $300 \mathrm{~cm}$ guide wire was introduced through the right brachial artery, moved across the aortic arch through the brachiocephalic artery, and inserted downstream to the femoral artery. The distal end of the wire was caught at the femoral artery cutdown. The guide wire was kept under continuous strain by traction at both ends of brachial and femoral access to overcome any possible tortuosity of the aortofemoral 


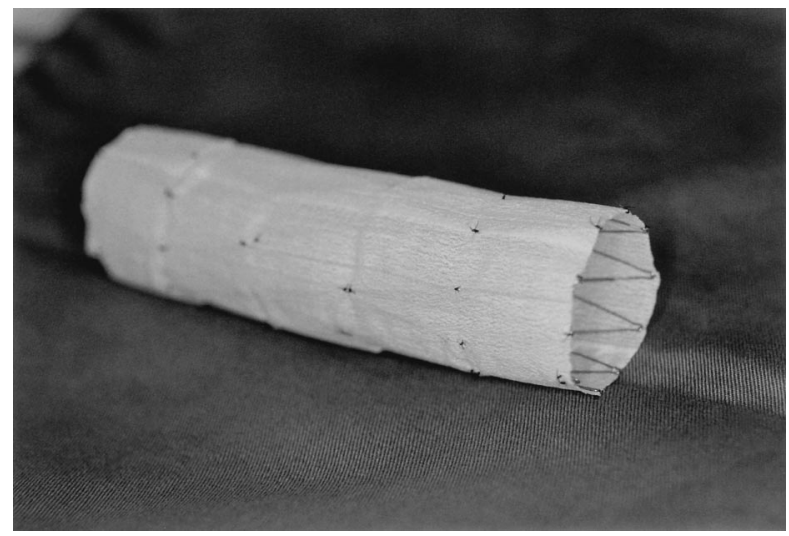

Fig. 3. Self-expanding $Z$ stents covered with ultrathin woven polyester fabric graft.

route when advancing the catheter ("tug of wire" method). An 18F PTFE sheath was introduced into the descending thoracic aorta over the guide wire with the tug of wire technique. After the proper position at which the permanent stent graft should be deployed was determined by perioperative angiography, the Retriever was compressed into and pushed to the end of a sheath by the pushing rod. When the Retriever was released from the sheath, it was allowed to expand by withdrawing the sheath while the pushing rod was held at the same position. The Retriever was placed in the aneurysm, extending to both cephalic and caudal necks, for at least 20 minutes and then retracted into the sheath by pulling on the pushing rod (Fig. 2).

The stent graft for permanent use was constructed of a self-expanding Gianturco Z stent (GZV; Cook Inc., Bjaeverskov, Denmark) covered with a seamless tube graft of ultrathin-wall woven polyester fabric $0.1 \mathrm{~mm}$ in thickness (Intervascular Inc., Clearwater, Fla.; Fig. 3). In patients in whom the temporary predeployment test by the Retriever was successful, permanent stent graft deployment was undertaken with nearly the same procedure described previously. Before expansion of the stent graft in the aorta, mean arterial pressure was decreased to approximately $70 \mathrm{~mm} \mathrm{Hg}$ through deep anesthesia and nifedipine administration.

ESCP monitoring was performed with a Neuropack System (MEB-5304; Nihon Kohden, Tokyo, Japan) according to the direct spinal cord stimulation method reported by Matsui and coworkers. ${ }^{7}$ In brief, two sets of five polar electrodes(IMC-KG-105; Inter Medical, Nagoya, Japan) were placed in the epidural space at the level of T-12 to L-1 for electrical stimulation and C-7 to $\mathrm{T}-1$ for potential recording the day before the stent graft operation. A current pulse of $0.2 \mathrm{msec}$ duration with $2 \mathrm{~Hz}$ frequency stimulation was applied with less than $5 \mathrm{~mA}$ intensity. The evoked potentials were averaged 50 times to eliminate electrical noise. Amplitude and latency of ESCP were measured before and every 3 minutes while the Retriever was in place and were monitored after permanent stent grafting until the next day.

\section{before Retriever \\ (BP: 124/64)}

\section{6 minutes \\ (BP: 134/60)}

\section{2 minutes \\ (BP: 130/58)}

\section{8 minutes}

(BP: 126/57)

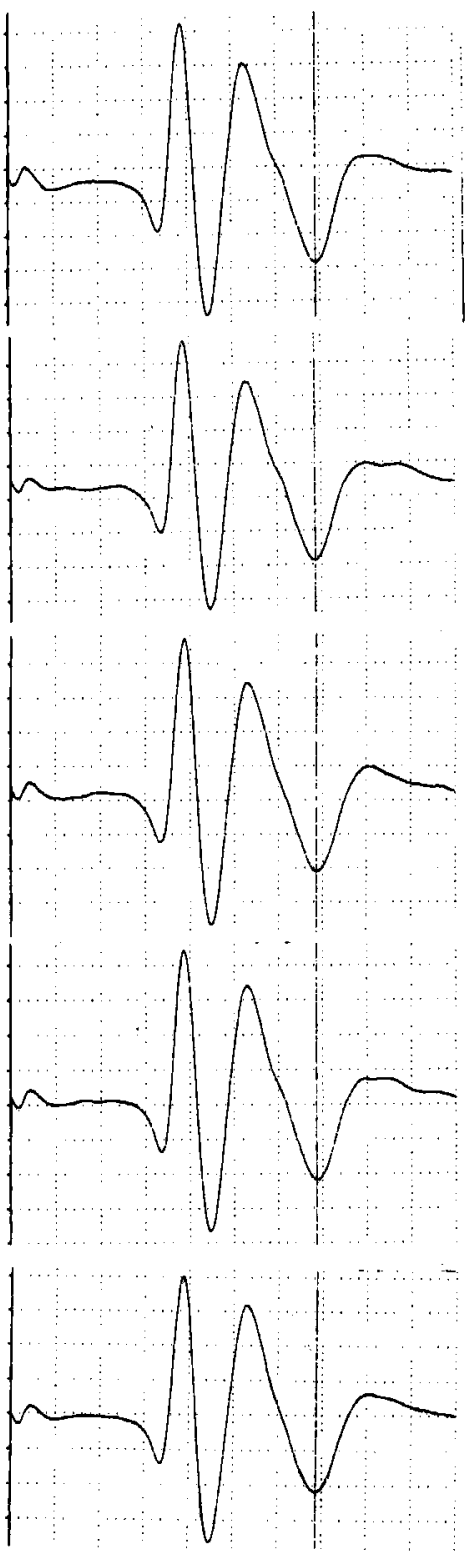

Fig. 4. No changes in amplitude and latency of ESCP records were observed before and during Retriever placement in patient 2. BP, Mean systemic arterial pressure in millimeters of mercury.

\section{Results}

Twenty-one aneurysms were detected in the descending aorta in 16 patients. They were located on the distal (T-8 to T-11) and middle (T-6 to T-9) segment of the descending aorta in 12 and five patients, respectively. Two isolated aneurysms (proximal and distal descending) and three isolated aneurysms (proximal, middle, and distal) were ob- 
Table I. Configuration of aneurysms, ESCP changes during Retriever placement, and postoperative results

\begin{tabular}{|c|c|c|c|c|c|c|c|}
\hline \multirow[b]{2}{*}{ Patient No. } & \multirow[b]{2}{*}{ Age (yr) } & \multirow{2}{*}{$\begin{array}{c}\text { Thoracic aneurysm } \\
\text { type and location }\end{array}$} & \multirow[b]{2}{*}{ ESCP change* } & \multicolumn{2}{|c|}{ Stent graft } & \multirow{2}{*}{$\begin{array}{c}\text { Neurologic } \\
\text { deficits }\end{array}$} & \multirow[b]{2}{*}{ Prognosis } \\
\hline & & & & Length $(\mathrm{mm})$ & Leak & & \\
\hline \multicolumn{8}{|l|}{ Nondissecting } \\
\hline 1 & 71 & Distal (T9-T10) & None & 50 & None & None & Good \\
\hline 2 & 58 & Distal (T8-T10 & None & 75 & None & None & Good \\
\hline 3 & 66 & Distal (T9-T11) & None & 125 & None & None & Good \\
\hline 4 & 62 & Distal (T9-T11) & None & 100 & None & None & Good \\
\hline 5 & 70 & Middle (T6-T9) & None & 175 & None & None & Good \\
\hline \multirow[t]{2}{*}{6} & 63 & Proximal & - & 50 & None & & \\
\hline & & Distal (T10-T11) & None & 75 & None & None & Good \\
\hline \multirow[t]{3}{*}{7} & 50 & Proximal & - & 100 & None & & \\
\hline & & Middle (T6-T8) & None & 75 & None & None & Good \\
\hline & & Distal (T9-T10) & None & 50 & None & & \\
\hline 8 & 77 & Distal (T9-T10) & None & 75 & None & None & Good \\
\hline \multirow[t]{2}{*}{9} & 74 & Proximal & - & 75 & None & & \\
\hline & & Distal (T8-T10) & None & 100 & None & None & Good \\
\hline 10 & 66 & Distal (T8-T10) & None & 100 & None & None & Good \\
\hline \multirow{2}{*}{11} & 71 & Proximal & & 75 & & & \\
\hline & & Distal (T9-T10) & None & 75 & None & None & Good \\
\hline \multicolumn{8}{|c|}{ Dissecting (Stanford B) } \\
\hline 12 & 59 & Middle (T7-T8)† & None & 75 & None & None & Good \\
\hline 13 & 76 & Distal $(\mathrm{T} 7-\mathrm{T} 10) \dagger$ & None & 125 & None & None & Good \\
\hline 14 & 64 & Distal (T8-T9) $\dagger$ & None & 75 & None & None & Good \\
\hline 15 & 60 & Middle (T6-T8)† & None & 75 & None & None & Good \\
\hline 16 & 34 & Middle (T7-T8) & None & 50 & None & None & Good \\
\hline
\end{tabular}

*Change in ESCP during temporary deployment of retrievable stent graft. $\dagger$ Ulcerlike projection.

$\ddagger$ Entry.

served in three patients and one patient, respectively. An $18 \mathrm{~F}$ delivery sheath was inserted at the femoral artery cutdown and introduced into the aorta, and extending both above and below it, by the tug of wire guiding technique in all patients. The Retriever was placed temporarily for at least 20 minutes in 17 aneurysms (12 distal, five middle of the descending aorta). Angiograms taken immediately after Retriever placement revealed delayed minor perigraft leakage. In six aneurysms more than $80 \mathrm{~mm}$ in length, the Retriever was placed at three consecutive and immediately adjacent sites (proximal neck, aneurysm, distal neck) to overcome lack of effective graft length for interruption of the intercostal arteries. Whether patent intercostals were present and covered by the Retriever was unclear.

ESCP monitoring was successfully carried out in all patients. There were narrow fluctuations in amplitude of ESCP within 5\% of the initial level, but no delay in latency from measurements taken every 3 minutes during approximately 20 minutes of Retriever placement with respect to the preinsertion baseline value (Fig. 4).

After withdrawal of the Retriever, the permanent stent graft was successfully deployed in all aneurysms. The length of the stent graft was decided according to that of the aneurysm, including its necks, measured by preoperative digital subtraction angiography and contrast-enhanced computed tomographic scan, and consequently varied from 50 to $175 \mathrm{~mm}$ ( $85 \mathrm{~mm}$ on average). All aneurysms located not only in the middle and distal but also proximal descending aorta were excluded immediately after stent grafting (Table I). The amplitude of ESCP decreased by more than $30 \%$ of the initial level when mean systemic arterial pressure was intentionally lowered below $70 \mathrm{~mm} \mathrm{Hg}$ to reduce migration of stent graft during its expansion (Fig. 5). There were no changes in ESCP from deployment of the permanent stent graft through the next day in all patients.

No endoleaks were revealed within aneurysms by postoperative digital subtraction angiography (Fig. 6) or enhanced computed tomography (Fig. 7). There were no neurologic deficits after patients awakened from anesthesia nor were there fatal complications related to the procedure during the follow-up period in any patient (Table I). 


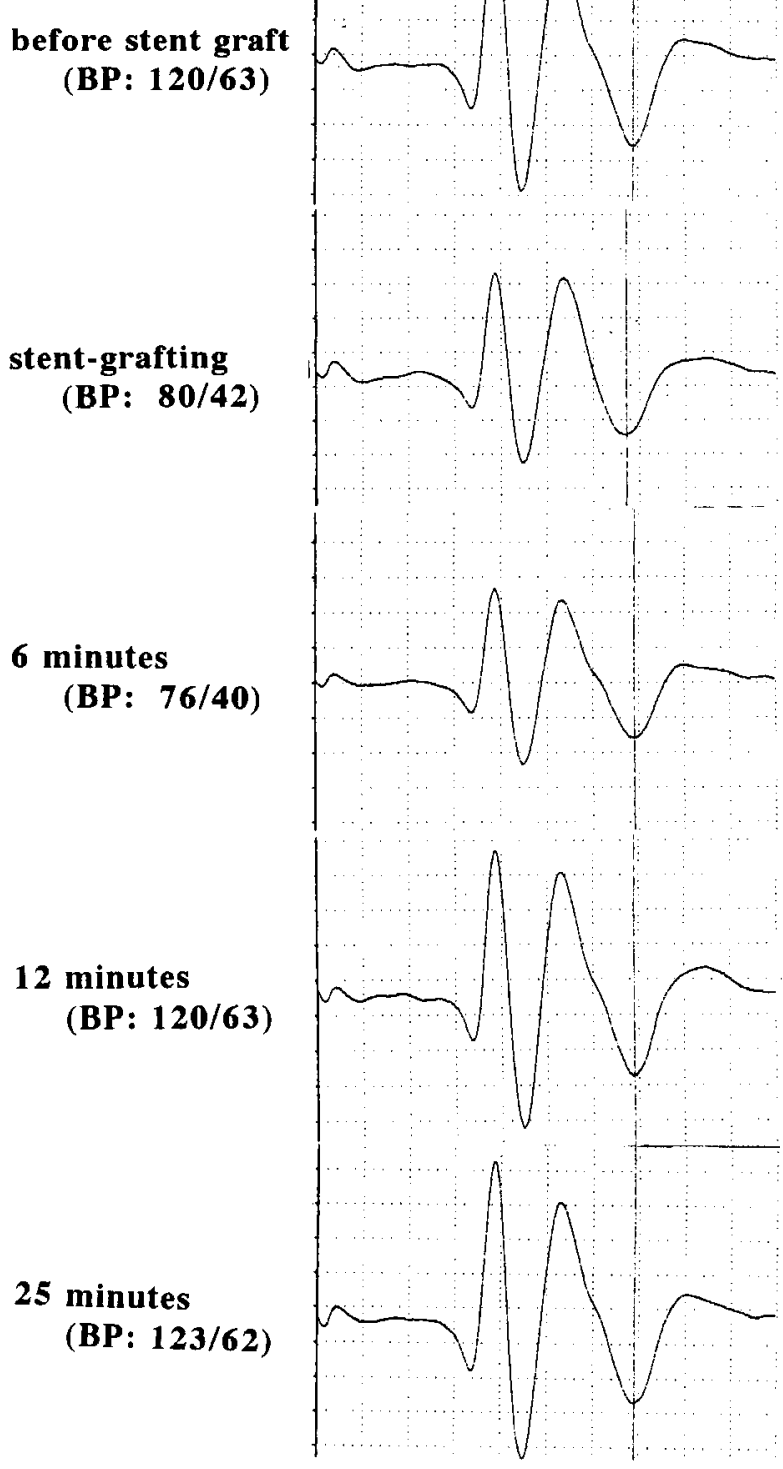

Fig. 5. In patient 2, amplitude of ESCP decreased to $40 \%$ of initial level when mean systemic arterial pressure $(B P)$ was decreased below $70 \mathrm{~mm} \mathrm{Hg}$ for 6 minutes, then recovered. There were no changes in ESCP after deployment of permanent stent graft.

\section{Discussion}

Paraplegia is a serious complication encountered in aortic operations. The risk of ischemic spinal cord injury is higher for patients with thoracic aortic aneurysm who require replacement of a long segment of the descending thoracic aorta or prolonged aortic crossclamp time. The arteries of Adamkiewicz

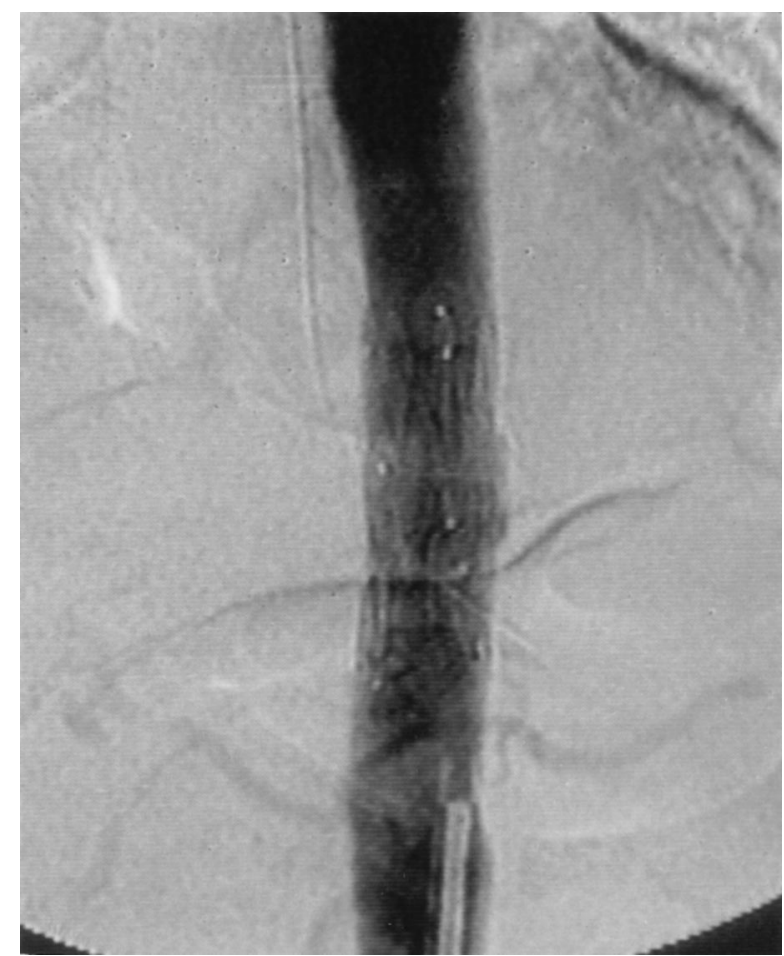

Fig. 6. Digital subtraction angiography of aneurysm excluded by stent graft deployment in patient 2 .

(the great radicular arteries) frequently (in approximately $81 \%$ of cases) originate from an intercostal branch between T- 8 and L-2 and provide the major blood flow to the anterior spinal artery in the region of the distal spinal cord. ${ }^{8}$ Prolonged interruption of blood supply to this branch may result in spinal cord ischemia and irreversible paraplegia. Despite various measures for intraoperative spinal cord protection, including cerebrospinal fluid drainage, systemic deep hypothermia, distal aortic perfusion with partial bypass, or reattachment of the critical intercostal arteries, the incidence of spinal cord injury was $4.5 \%$ to $21 \%$ among patients with descending thoracic or thoracoabdominal aortic aneurysm.., 10

Concerning endovascular stent graft repair of thoracic aortic aneurysm, it is possible that stent graft deployment could induce interruption of the blood flow in critical intercostal arteries originating from the descending aorta, especially in the middle or distal segment (T-8 or below), including the aneurysmal region and its proximal and distal necks. It is therefore essential to determine the critical arteries precisely and avoid occlusion of blood flow to reduce the risk of spinal cord ischemia. Detection of the critical segmental arteries has been per- 


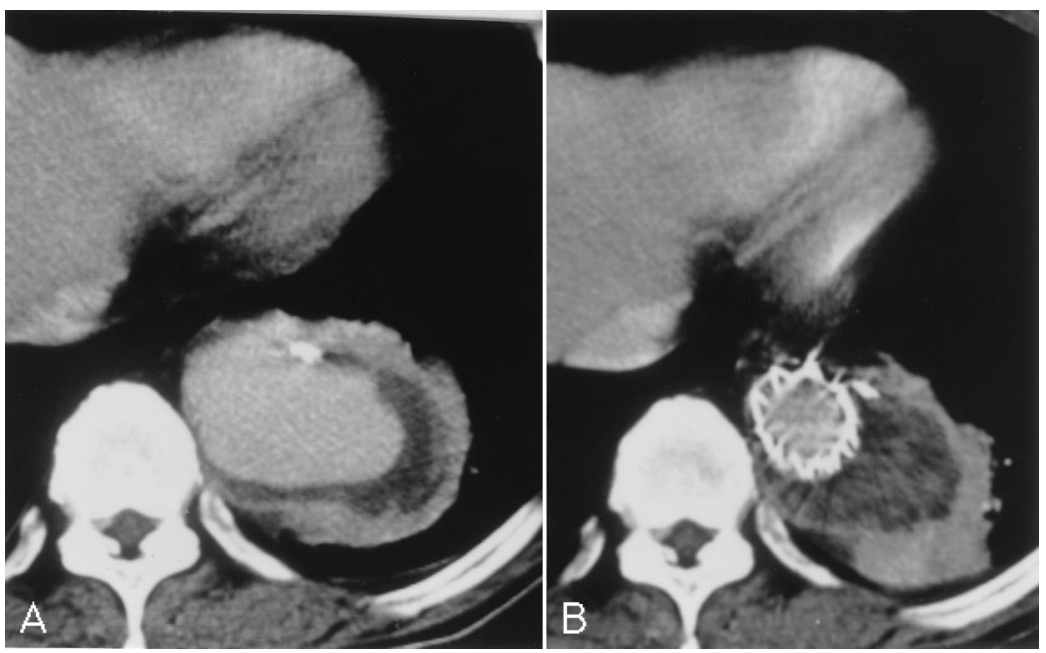

Fig. 7. A, Enhanced computed tomography before stent graft deployment in patient 2. B, After placement of stent graft, no endoleaks were confirmed in the aneurysm.

formed by preoperative selective angiography ${ }^{11}$ or intraoperative injection of a hydrogen-saturated saline solution marker. ${ }^{12}$ However, it is difficult to prove deterioration of spinal cord ischemia by these morphologic examinations. Somatosensory evoked potentials ${ }^{13}$ and $\mathrm{ESCP}^{14}$ have been reported to be reliable methods for the detection of ischemia actually occurring in the spinal cord, although motor system disturbance is not sensitively reflected by either method.

In this study a predeployment test with a novel retrievable stent graft, the Retriever, was performed to detect spinal cord ischemia by temporary interruption of blood flow into the intercostal arteries and the aneurysmal sac, which would be permanently covered with the stent graft later. It was easy to introduce the Retriever into the descending aorta through an $18 \mathrm{~F}$ sheath and allow it to expand in the target region. Withdrawal of the Retriever was successfully performed in all cases. There was no change in ESCP during the predeployment test except for narrow fluctuation in amplitude. Decrease in ESCP amplitude indicates spinal cord ischemia, regardless of whether the latency is delayed. ${ }^{15}$ The ESCP amplitude decreased by more than $30 \%$ of the initial levels when the mean systemic arterial pressure decreased below $70 \mathrm{~mm} \mathrm{Hg}$ and then increased even if the stent graft was not yet deployed in the aorta. A change in ESCP amplitude indicated a decrease in arterial pressure to the critical ischemic level. These results indicate that ESCP is a sensitive and reliable monitor of ischemia in the spinal cord. Although minor leakage was observed at both ends of the Retriever because the expanded PTFE sheet that lines the stent frame did not adhere completely to the aortic wall, extreme delay in filling of the aneurysm with contrast medium on angiography would suggest that decompression of the aneurysm and interruption of the intercostal arteries induced spinal cord ischemia of a degree that could be detected precisely by ESCP monitoring.

There has been no accurate analysis of complications related to neurologic deficits observed in stent graft aneurysm repair because the amount of clinical experience of stent grafting in the thoracic aorta is still limited. In the Stanford study reported in 1996, Mitchell and associates ${ }^{5}$ stated that paraplegia observed in two $(5 \%)$ of 39 patients was not caused by interruption of the critical intercostal artery but by distal hypoperfusion or atheroembolism during stent grafting. In this study, however, patients with aneurysm extending over the critical intercostal arteries were excluded from the indications of stent graft repair. On the contrary, our report describes 49 cases with thoracic aortic aneurysm, including 16 critical cases $(33 \%)$ in which the aneurysm was partially or entirely located between T-8 and T-11, where the Adamkiewicz great radicular artery frequently originates. ${ }^{8}$ The ratio of patients at critical risk of spinal cord ischemia in this series seems sufficiently high to suggest a high rate of possibility of paraplegia after stent graft-induced spinal cord ischemia, and the Stanford experience and that of Borst and coworkers ${ }^{9}$ suggest that this serious complication would probably have occurred in at least 
one patient in this series. Consequently, it is noteworthy that there was no paraplegia caused by permanent stent graft placement in this study, suggesting the possible reliability of the predeployment test by the retrievable stent graft under ESCP monitoring for the detection of any ischemic change caused by stent grafting. If complete disappearance of ESCP or a significant decrease in amplitude ( $>30 \%$ of the initial level) is observed during the predeployment test, surgical aneurysm repair with reattachment of the critical intercostal arteries rather than endovascular stent grafting is mandatory. Of course, further experience with more cases is necessary before meaningful statistics can be generated.

Although induction distal thromboembolism could be almost completely avoided by application of our tug of wire technique in which the catheter is advanced into the tortuous and sclerotic arteries over the guide wire, the risk of atheroembolism in the critical arteries that supply blood flow to the spinal cord, with resulting paraplegia, still remains to a certain degree because of the expansion and retraction of the Retriever. Development of a removable stent graft for permanent use would therefore help eliminate the risk of endoluminal laceration of the aorta without the necessity of our predeployment test.

Only when the risk of neurologic complications has been reduced to negligible proportions will endovascular stent grafting truly be recognized as a valid catheter-based minimally invasive surgical strategy for thoracic aortic aneurysms. Further investigation is necessary to clarify graft durability, aneurysmal neck enlargement, and the fate of the excluded aneurysm sac for long follow-up periods.

We are indebted to Professor J. Patrick Barron of the International Medical Language Center of Tokyo Medical College for his review of the manuscript.

\section{REFERENCES}

1. Hayashi J, Eguchi S, Yasuda K, Komatsu S, Tabayashi K, Masuda M, et al. Operation for non dissecting aneurysm in the descending thoracic aorta. Ann Thorac Surg 1997;63: 93-7.

2. Verdant A, Cossette R, Page A, Baillot R, Dontigny L, Page
P. Aneurysms of the descending thoracic aorta: three hundred sixty-six consecutive cases resected without paraplegia. J Vasc Surg 1995;21:385-91.

3. Parodi JC, Palmaz JC, Barone HD. Transfemoral intraluminal graft implantation for abdominal aortic aneurysms. Ann Vasc Surg 1991;5:491-9.

4. Dake MD, Miller DC, Semba CP, Walker PJ, Liddell RP. Transluminal placement of endovascular stent-grafts for the treatment of descending thoracic aortic aneurysms. N Engl J Med 1994;331:1729-34.

5. Mitchell RS, Dake MD, Semba CP, Fogarty TJ, Zarins CK, Liddell RP, et al. Endovascular stent-graft repair of thoracic aortic aneurysms. J Thorac Cardiovasc Surg 1996;111:105462.

6. Wright $\mathrm{KC}$, Wallace $\mathrm{S}$, Chamsangavej $\mathrm{C}$, Carrasco $\mathrm{CH}$, Gianturco C. Percutaneous endovascular stents; an experimental evaluation. Radiology 1985;156:156-69.

7. Matsui Y, Goh K, Shiiya N, Murashita T, Miyama M, Ohba $\mathrm{J}$, et al. Clinical application of evoked spinal cord potentials elicited by direct stimulation of the cord during temporary occlusion of the thoracic aorta. J Thorac Cardiovasc Surg 1994;107:1519-27.

8. Kieffer E, Richard T, Chiras J, Godet G, Cormier E. Preoperative spinal cord arteriography in aneurysmal disease of the descending thoracic and thoracoabdominal aorta: preliminary results in 45 patients. Ann Vasc Surg 1989;3:34-46.

9. Borst HG, Jurmann M, Buhner B, Laas J. Risk of replacement of descending aorta with a standardized left heart bypass technique. J Thorac Cardiovasc Surg 1994;107:12633.

10. Cox GS, O'Hara PJ, Hertzer NR, Piedmonte MR, Krajewski LP, Beven EG. Thoracoabdominal aneurysm repair: a representative experience. J Vasc Surg 1992;15:780-8.

11. Williams GM, Perler BA, Burdick JF, Osterman FA Jr, Mitchel S, Merine D, et al. Angiographic localization of spinal cord blood supply and its relationship to postoperative paraplegia. J Vasc Surg 1991;13:23-35.

12. Svensson LG, Patel V, Crawford ES. Preliminary report of localization of spinal cord blood supply by hydrogen during aortic operation. Ann Thorac Surg 1990;49:528-36.

13. Laschinger JC, Cunningham JN Jr, Catinella FP, Nathan IM, Knopp EA, Spencer FC. Detection and prevention of intraoperative spinal cord ischemia after cross-clamping of the thoracic aorta: use of somatosensory evoked potentials. Surgery 1982;92:1109-17.

14. Shiiya N, Yasuda K, Matsui Y, Sakuma M, Sasaki S. Spinal cord protection during thoracoabdominal aortic aneurysm repair: results of selective reconstruction of the critical segmental arteries guided by evoked spinal cord potential monitoring. J Vasc Surg 1995;21:970-5.

15. Yamamoto N, Takano H, Kitagawa H, Kawaguchi Y, Tsuji $\mathrm{H}$, Uozaki Y. Monitoring for spinal cord ischemia by use of the evoked spinal cord potentials during aortic aneurysm surgery. J Vasc Surg 1994;20:826-33. 